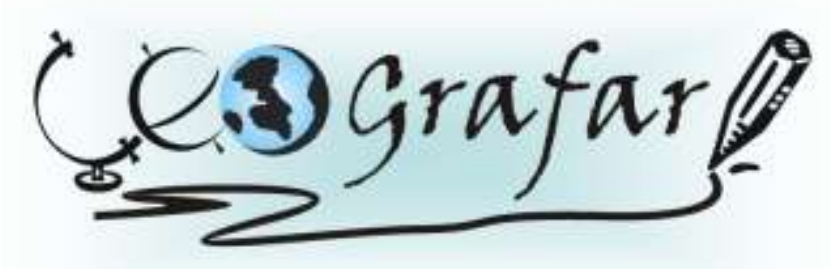

Revista Eletrônica do Programa de Pós-Graduaçāo em Geografla - UFPR

\title{
PRECIPITAÇÕES PLUVIAIS EXTREMAS EM LONDRINA - PR
}

\author{
GLAUCO MARIGHELLA FERREIRA DA SILVA ${ }^{1}$ \\ PAULO HENRIQUE CARAMORI ${ }^{2}$ \\ ROGÉRIO TEIXEIRA DE FARIA ${ }^{3}$
}

\begin{abstract}
RESUMO
O presente trabalho tem por intuito estimar as máximas pluviométricas para o município de Londrina - PR, através da identificação dos maiores eventos de precipitação diária ocorridos (eventos extremos) em cada ano da série histórica em análise, auxiliando no planejamento e na tomada de decisão principalmente quanto a dimensionamento de estruturas e prevenção de desastres. É de conhecimento comum de que as chuvas em grandes proporções podem gerar transtornos, entretanto, não existem dados atualizados sobre as precipitações máximas estimadas para o município de Londrina e desta forma o trabalho vem a contribuir para que os profissionais e o serviço público possam prevenir a população de eventos inesperados. Os dados utilizados foram coletados de 1961 a 2010 nas estações meteorológicas do Instituto Nacional de Meteorologia (INMET) e Agronômico do Paraná (IAPAR), localizado no município de Londrina. Para a estimativa de precipitações máximas utilizou-se o método de distribuição de Gumbel, juntamente com a equação geral de duração de precipitações máximas de Holtz (1966). Os resultados possibilitam melhorar o dimensionamento de estruturas nos meios rural e urbano, reduzindo impactos de eventos extremos.
\end{abstract}

Palavras-chave: Distribuição de Gumbel; precipitações extremas; máximas pluviométricas em Londrina.

\footnotetext{
${ }^{1}$ Graduando do curso de Geografia da Universidade Estadual de Londrina. Membro Dirigente do CREAjr-PR. Bolsista CNPq - IAPAR. E-mail: glaucomarighella@hotmail.com.

${ }^{2}$ Eng. Agrônomo, PhD Agrometeorologia. Pesquisador do Instituto Agronômico do Paraná (IAPAR) ebolsista do CNPq. E-mail: caramori@iapar.br.

3 Eng. Agrônomo, PhD Engenharia Agrícola. Pesquisador aposentado do Instituto Agronômico do Paraná (IAPAR), atualmente na UNESP-Campus de Jaboticabal, bolsista do CNPq. E-mail: rtdefaria@gmail.com.
} 


\title{
EXTREME PLUVIAL PRECIPITATION IN LONDRINA - PR
}

\begin{abstract}
The purpose of this paper is to estimate the maximum rainfall for the municipality of Londrina - PR, by identifying the major daily precipitation events occurred (extreme events) in each year of the series under analysis, assisting in planning and decision making particularly regarding the design of structures and disaster prevention. It is common knowledge that rainfall can cause major disorders; however there are no current data on the estimated maximum rainfall for the municipality of Londrina, so the work is to contribute to the professional and public service to prevent the population of unexpected events. The estimates ofreturn periods of precipitations were performed with the Gumbel distribution, together with the general equation for the duration of precipitations of Holtz (1966). The data were collected from 1961 to 2010 at the weather stations of the National Institute of Meteorology (INMET) and the Agronomic Institute of Paraná (IAPAR), located in Londrina. The results help to improve the design of structures in rural and urban areas, reducing impacts of extreme events.
\end{abstract}

Key-words: Gumbel distribution, extreme rainfall, maximum rainfall in Londrina.

\section{INTRODUÇÃO}

A cultura humana está intimamente ligada aos fenômenos climáticos. As chuvas são essenciais para a manutenção da vida e fator eficaz para o sucesso das safras agrícolas. Entretanto, chuvas em grande quantidade podem gerar problemas relacionados a inundações, perda de safras, enchentes, destruição da infraestrutura urbana e rural, riscos de deslizamentos e consequentemente mortes.

Grandes eventos pluviais podem gerar saturação do solo, levando ao aumento do fluxo superficial e causar perda de solo por enxurrada, acúmulo superficial de água, gerando prejuízos para toda a sociedade.

O estudo de eventos extremos se torna essencial para construção de obras de engenharia que suportem também grandes chuvas, pois, apesar de serem excepcionais, tem altas probabilidades de gerar sérios problemas devido a falhas na estimativa de valores críticos nos projetos, seja para o agricultor, cuja produção depende das condições hídricas da sua lavoura, seja para o homem da cidade, que convive com distúrbios gerados por fortes chuvas. 
Visando todos esses problemas gerados por excesso de chuva, principalmente em eventos seguidos ou em curtos períodos de tempo, o presente trabalho vem com o intuito de identificar os eventos pluviais extremos e estimar 0 período de retorno dos mesmos, além de gerar parâmetros estatísticos de probabilidade de limites de chuva a partir de dados dos últimos 50 anos (1961-2010) em Londrina-PR.

\section{MÉTODO}

A quantidade de chuva depende de diversos fatores climáticos, destacandose correntes e massas de ar, localização geográfica, condições de estabilidade e relevo.

Sendo assim, esses diversos fatores se combinam ao longo dos anos e criam uma dinâmica complexa, cuja compreensão vem sendo exaustivamente buscada pela ciência. Sua regularidade e as interligações entre as diferentes forças da natureza ainda não são totalmente compreendidas, e tão pouco existem séries de dados meteorológicos longas o suficiente para representar os ciclos climáticos. Os eventos climáticos são relacionados principalmente às estações do ano, não se considerando a variação que a mesma pode ter dentro de períodos maiores, chegando alguns autores a afirmarem que esses ciclos podem ser mais longos que os próprios registros humanos (SANT'ANNA NETO, 2007).

As precipitações pluviais extremas estão ligadas principalmente ao aquecimento do ar úmido próximo ao solo, que, ao se perturbar sua estabilidade, sofre rápida ascensão, fazendo com que se precipitem enormes quantidades de água em um curto período de tempo, principalmente durante o verão no Centro-Sul do Brasil. Essas chuvas, geralmente, têm grande intensidade e pequena duração e podem ocasionar inundações (TUCCI, 2000).

Esses grandes eventos pluviais afetam as atividades humanas, pois geram inundações, desmoronamentos, enchentes, perda de lavouras, destruição de infraestrutura e mortes. O conhecimento dos limites máximos de chuva é essencial para dimensionar a capacidade de suporte de estruturas de contenção, escoamento e drenagem e assim minimizar os danos. 
O aumento do fator de segurança das obras de engenharia consequentemente provoca aumento dos valores das construções, entretanto o risco de falhas se torna menor e o tempo de vida da mesma maior. Desta forma se torna essencial conhecer a quantidade máxima que o projeto terá que suportar ao longo dos anos.

Conforme Farias (1990), as formas diretas de obtenção de dados a respeito de vazão e pluviosidade são mais confiáveis, através de medições diárias, entretanto elas são mais caras e nem sempre é possível obter grandes séries de dados contínuos dependendo da região, além do tempo para obtenção ser muito longo. A grande vantagem de se utilizar séries de dados de precipitações máximas em estudos de chuva-vazão de bacias hidrográficas é a maior disponibilidade de séries históricas de precipitação em comparação com dados de vazão (TUCCl, 2000). Por isso as formas indiretas são as mais utilizadas, por meio do uso de equações matemáticas que estimam os limites dos índices de pluviosidade e vazão para aplicações no dimensionamento de obras hidráulicas (MELLO; SILVA, 2005).

Apesar de não retratarem a realidade, já que não se pode afirmar que uma mesma quantidade de chuva em certo período do ano é equivalente a uma chuva alguns meses mais tarde, pois não necessariamente estão ligados às mesmas condições atmosféricas, os modelos probabilísticos de distribuição de valores extremos são válidos e muito empregados na hidrologia (NAGHETTINI; PINTO, 2007).

A utilização de linguagem matemática para eventos pluviais se justifica já que a mesma gera a capacidade de prever e estimar a quantidade, a duração e a intensidade, auxiliando no cotidiano e no planejamento, principalmente quando se trata de precipitações pluviais extremas.

Os resultados do presente trabalho foram gerados por meio da análise de diários de precipitação coletados nas estações meteorológicas do Instituto Nacional de Meteorologia (INMET) de 1961 a 1975 e do Instituto Agronômico do Paraná (IAPAR), de 1976 a 2010, ambas localizadas no município de Londrina, a 3km de distância em linha reta. A série de dados corresponde a 50 anos de registros ininterruptos, quantidade acima do recomendado pela Organização Meteorológica Mundial (OMM), que recomenda uma série de pelo menos 30 anos para uma análise consistente. Antes de se utilizar os dados, foram realizados testes de 
homogeneidade das séries, conforme WMO (1983), verificando-se que os mesmos podem ser analisados como uma série contínua. Desta forma, os registros foram organizados em uma planilha eletrônica, verificados e consistidos quanto a possíveis erros de digitação e posteriormente analisados.

Os cálculos foram realizados com a aplicação da metodologia de Kessler \& Raad (1980 apud FARIA e WAGNER, 1990). Deste modo, foram considerados períodos de precipitação de 1 a 6 dias ( $\mathrm{t}=1,2,3,4,5$ e 6$)$. Assim, a pluviosidade para 1 dia foi obtida pelo registro diário da estação, enquanto de 2 a 6 dias a quantidade de chuva foi a soma dos dias adjacentes (2 dias de chuva são equivalentes à soma do dia anterior com o dia em questão, 3 dias equivale à soma dos dois dias anteriores e o dia em questão, etc.). Dos dados obtidos para cada período em análise, foram selecionados os maiores eventos pluviométricos dos anos, que foram tratados como as precipitações pluviais extremas em Londrina.

FIGURA 1 - LOCALIZAÇÃO DE LONDRINA NO NORTE PARANAENSE

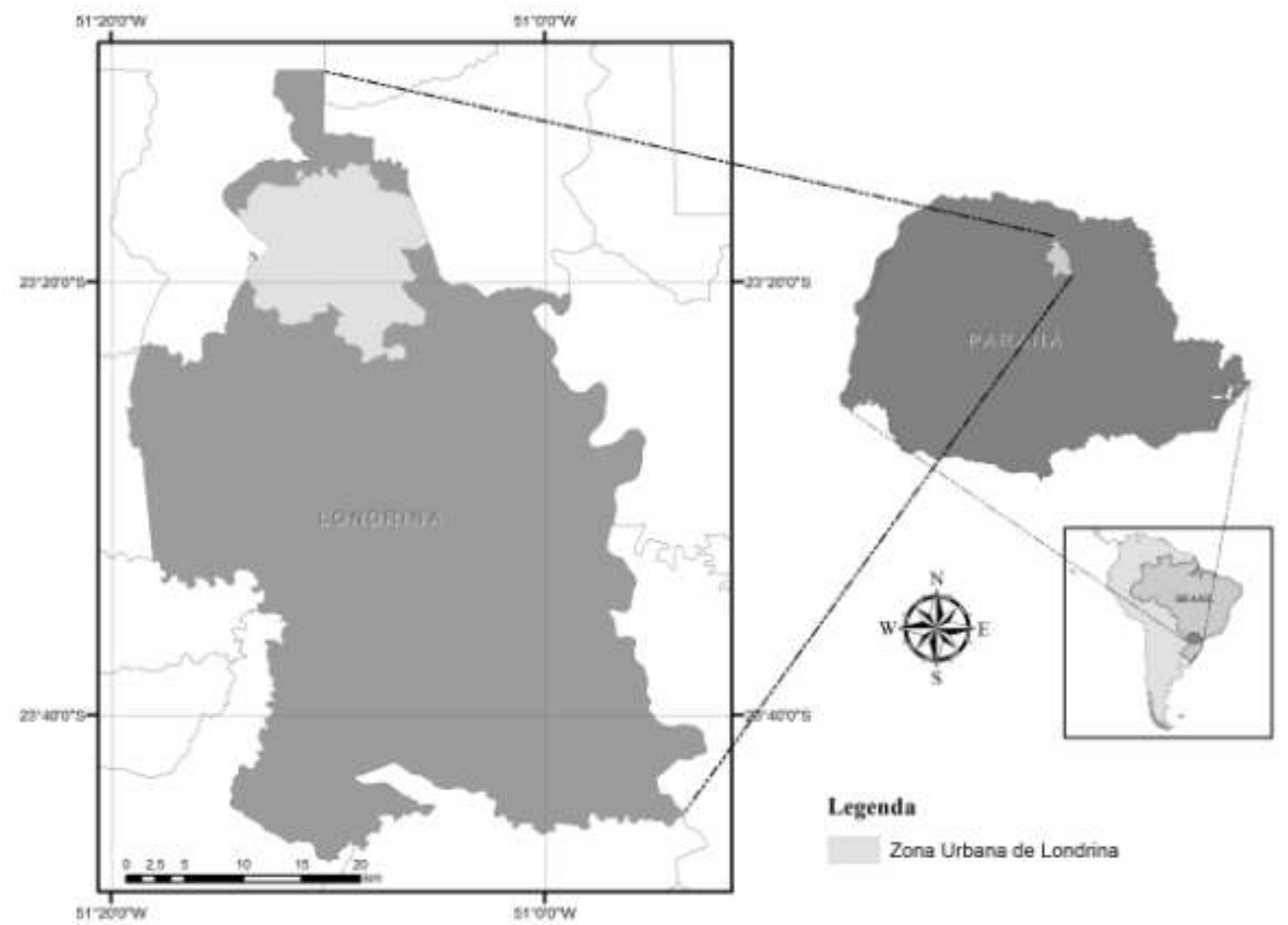

Elaboração: SILVA, G. M. F. 
Esses valores extremos foram ordenados em valores decrescentes para cada período, sendo o maior valor denotado como 1. O resultado correspondeu às precipitações máximas observadas com durações de um a seis dias.

Assim, determinaram-se as precipitações máximas esperadas e os períodos de recorrência observados. Para a determinação do tempo de recorrência, utilizouse a distribuição de Gumbel (ou distribuição de extremos do tipo I), que consiste na fórmula básica (TUCCI, 2000):

$$
P(X \leq x)=\exp (-\exp (-y))
$$

em que $\mathbf{P}$ a probabilidade de um dado valor ser igualado ou excedido e y é a variável reduzida dada por:

$$
\begin{aligned}
& \mathbf{y}=\left(\mathbf{x}-\mathbf{X}_{\mathbf{f}}\right) \frac{s_{n}}{s_{x}} \\
& \mathbf{X}_{\mathrm{f}}=\bar{x}-S_{\mathbf{x}}\left(\frac{\overline{Y_{n}}}{s_{n}}\right)
\end{aligned}
$$

em que xf é a moda dos valores extremos, Sn é o desvio padrão da variável reduzida $\mathbf{Y}, \mathbf{S x}$ é o desvio padrão da variável $\mathbf{x}$, e $\overline{\boldsymbol{x}}$ e $\overline{\boldsymbol{y}}$, as médias das variáveis $\mathbf{x}$ e $\mathbf{y}$, respectivamente.

Os valores $\mathbf{Y}_{\mathbf{n}}$ e $\mathbf{S}_{\mathbf{n}}$ são obtidos em função do número de dados (Tabela 1).

TABELA 1 - VALORES TABELADOS DAS VARIÁVEIS REDUZIDAS Y Y $\mathrm{N}_{N} \mathrm{~S}_{\mathrm{N}} \mathrm{E}$ DESVIO PADRÃO DA VARIÁVEL REDUZIDA Y, EM FUNÇÃO DO NÚMERO $n$ DE VARIÁVEIS.

\begin{tabular}{lll}
\hline $\mathrm{n}$ & $\mathrm{Y}_{\mathrm{n}}$ & $\mathrm{S}_{\mathrm{n}}$ \\
\hline 20 & 0,52 & 1,06 \\
30 & 0,54 & 1,11 \\
40 & 0,54 & 1,14 \\
50 & 0,55 & 1,16 \\
60 & 0,55 & 1,17 \\
70 & 0,55 & 1,19 \\
80 & 0,56 & 1,19 \\
90 & 0,56 & 1,20 \\
100 & 0,56 & 1,21 \\
150 & 0,56 & 1,23 \\
200 & 0,57 & 1,24 \\
$\infty$ & 0,57 & 1,28 \\
\hline
\end{tabular}

Fonte: (Villela, 1975). 
A probabilidade inversa, ou seja, a possibilidade do evento não ser igualado ou excedido é dado por:

$$
P(X \geq X)=1-P
$$

O tempo de recorrência $(\mathbf{T})$ corresponde a:

$$
\mathbf{T}=\frac{1}{1-P}
$$

Tendo-se as equações ajustadas à distribuição de Gumbel para cada tempo de duração, ajustou-se a equação geral de estimativa de precipitações máximas prováveis para períodos de retorno $T=2,5,10,20$ e 30 anos e de tempo de duração t = 1, 2, 3, 4, 5 e 6 dias de Holtz (1966 apud FARIA e WAGNER, 1990):

$$
\boldsymbol{H}=\boldsymbol{a} \cdot \boldsymbol{T}^{b} \cdot(\boldsymbol{t}-\boldsymbol{c})^{d},
$$

em que $\mathbf{H}$ representa a pluviosidade máxima estimada ou observada, $\mathbf{T}$ o tempo de recorrência calculado e t o número de dias de duração do evento pluvial. O ajuste desta equação foi realizado com o auxílio do software SigmaPlot@, utilizando procedimentos de ajuste de modelo de regressão não linear para obtenção dos coeficientes a, b, c e d.

\section{RESULTADOS E DISCUSSÕES}

As tabelas 2 a 7 apresentam as precipitações máximas observadas, Y a variável reduzida, $\mathrm{P}$ a probabilidade da precipitação não ser excedia, 1-P a probalidade de ser excedida. 
TABELA 2 - VALORES OBTIDOS PARA PRECIPITAÇÕES MÁXIMAS COM DURAÇÃO DE 1 DIA

\begin{tabular}{|c|c|c|c|c|c|c|}
\hline ANO & $\begin{array}{c}\text { Precipitação } \\
\text { em } 1 \text { dia }\end{array}$ & $Y$ & $\mathbf{P}$ & $(1-P)$ & $\begin{array}{l}\text { Período de } \\
\text { Recorrência } \\
\text { em anos }(T)\end{array}$ & $\begin{array}{l}\text { Ordem: } \\
1 \text { dia (r) }\end{array}$ \\
\hline 1997 & 161,0 & 4,295 & 0,986 & 0,014 & 73,864 & 1 \\
\hline 1984 & 151,2 & 3,802 & 0,978 & 0,022 & 45,313 & 2 \\
\hline 1992 & 142,7 & 3,375 & 0,966 & 0,034 & 29,723 & 3 \\
\hline 1991 & 119,1 & 2,188 & 0,894 & 0,106 & 9,424 & 4 \\
\hline 1989 & 117,1 & 2,087 & 0,883 & 0,117 & 8,572 & 5 \\
\hline 1977 & 116,3 & 2,047 & 0,879 & 0,121 & 8,254 & 6 \\
\hline 1993 & 113,5 & 1,906 & 0,862 & 0,138 & 7,239 & 7 \\
\hline 2010 & 108,2 & 1,639 & 0,824 & 0,176 & 5,668 & 8 \\
\hline 1994 & 106,4 & 1,549 & 0,809 & 0,191 & 5,224 & 9 \\
\hline 2007 & 104,8 & 1,468 & 0,794 & 0,206 & 4,861 & 10 \\
\hline 1982 & 102,0 & 1,328 & 0,767 & 0,233 & 4,294 & 11 \\
\hline 1975 & 98,9 & 1,172 & 0,734 & 0,266 & 3,753 & 12 \\
\hline 1985 & 98,2 & 1,136 & 0,725 & 0,275 & 3,642 & 13 \\
\hline 1996 & 96,1 & 1,031 & 0,700 & 0,300 & 3,333 & 14 \\
\hline 2009 & 96,0 & 1,026 & 0,699 & 0,301 & 3,319 & 15 \\
\hline 1974 & 92,8 & 0,865 & 0,656 & 0,344 & 2,909 & 16 \\
\hline 1972 & 85,0 & 0,472 & 0,536 & 0,464 & 2,155 & 17 \\
\hline 2005 & 84,2 & 0,432 & 0,522 & 0,478 & 2,094 & 18 \\
\hline 1987 & 83,2 & 0,382 & 0,505 & 0,495 & 2,021 & 19 \\
\hline 1988 & 83,0 & 0,372 & 0,502 & 0,498 & 2,007 & 20 \\
\hline 1964 & 82,8 & 0,362 & 0,498 & 0,502 & 1,993 & 21 \\
\hline 1998 & 82,8 & 0,362 & 0,498 & 0,502 & 1,993 & 22 \\
\hline 1962 & 82,4 & 0,342 & 0,491 & 0,509 & 1,966 & 23 \\
\hline 1978 & 81,4 & 0,291 & 0,474 & 0,526 & 1,900 & 24 \\
\hline 1963 & 81,0 & 0,271 & 0,466 & 0,534 & 1,874 & 25 \\
\hline 1980 & 80,0 & 0,221 & 0,448 & 0,552 & 1,813 & 26 \\
\hline 1968 & 78,7 & 0,155 & 0,425 & 0,575 & 1,739 & 27 \\
\hline 1990 & 77,8 & 0,110 & 0,408 & 0,592 & 1,690 & 28 \\
\hline 1961 & 77,0 & 0,070 & 0,394 & 0,606 & 1,649 & 29 \\
\hline 2002 & 77,0 & 0,070 & 0,394 & 0,606 & 1,649 & 30 \\
\hline 1969 & 76,6 & 0,050 & 0,386 & 0,614 & 1,629 & 31 \\
\hline 1973 & 76,6 & 0,050 & 0,386 & 0,614 & 1,629 & 32 \\
\hline 2001 & 76,4 & 0,040 & 0,382 & 0,618 & 1,619 & 33 \\
\hline 1976 & 75,0 & $-0,031$ & 0,357 & 0,643 & 1,554 & 34 \\
\hline 1983 & 74,8 & $-0,041$ & 0,353 & 0,647 & 1,545 & 35 \\
\hline 1999 & 74,8 & $-0,041$ & 0,353 & 0,647 & 1,545 & 36 \\
\hline 2003 & 73,8 & $-0,091$ & 0,334 & 0,666 & 1,502 & 37 \\
\hline 1965 & 73,4 & $-0,111$ & 0,327 & 0,673 & 1,486 & 38 \\
\hline 1970 & 73,2 & $-0,121$ & 0,323 & 0,677 & 1,478 & 39 \\
\hline 2000 & 68,9 & $-0,338$ & 0,246 & 0,754 & 1,327 & 40 \\
\hline 1995 & 68,8 & $-0,343$ & 0,244 & 0,756 & 1,324 & 41 \\
\hline 2004 & 68,8 & $-0,343$ & 0,244 & 0,756 & 1,324 & 42 \\
\hline 1981 & 65,9 & $-0,488$ & 0,196 & 0,804 & 1,244 & 43 \\
\hline 1966 & 65,2 & $-0,524$ & 0,185 & 0,815 & 1,227 & 44 \\
\hline 1986 & 62,5 & $-0,659$ & 0,145 & 0,855 & 1,169 & 45 \\
\hline
\end{tabular}




\begin{tabular}{ccccccc}
\hline $\mathbf{1 9 7 9}$ & 62,0 & $-0,685$ & 0,138 & 0,862 & 1,160 & 46 \\
$\mathbf{1 9 7 1}$ & 60,7 & $-0,750$ & 0,120 & 0,880 & 1,137 & 47 \\
$\mathbf{2 0 0 8}$ & 59,8 & $-0,795$ & 0,109 & 0,891 & 1,123 & 48 \\
$\mathbf{2 0 0 6}$ & 59,0 & $-0,836$ & 0,100 & 0,900 & 1,111 & 49 \\
$\mathbf{1 9 6 7}$ & 50,5 & $-1,263$ & 0,029 & 0,971 & 1,030 & 50 \\
\hline MĖDIA (x) & 86,55 & & & & & \\
DESVIO & & & & & & \\
PADRÃO & 23,061 & & & & & \\
(Sx) & & & & & & \\
Sn & 1,16 & & & & & \\
Yn & 0,55 & & & & & \\
Xf & 75,612 & & & & & \\
\hline
\end{tabular}

TABELA 3 - VALORES OBTIDOS PARA PRECIPITAÇÕES MÁXIMAS COM DURAÇÃO DE 2 DIAS

\begin{tabular}{|c|c|c|c|c|c|c|}
\hline ANO & $\begin{array}{l}\text { Precipitação } \\
\text { em } 2 \text { dias }\end{array}$ & $\mathbf{Y}$ & $\mathbf{P}$ & $(1-P)$ & $\begin{array}{l}\text { Período de } \\
\text { Recorrência } \\
\text { em anos }(T)\end{array}$ & $\begin{array}{l}\text { Ordem: } \\
2 \text { dias }(r)\end{array}$ \\
\hline 1989 & 212,40 & 3,87 & 0,98 & 0,02 & 48,55 & 1 \\
\hline 1992 & 185,20 & 2,88 & 0,95 & 0,05 & 18,35 & 2 \\
\hline 1997 & 182,30 & 2,78 & 0,94 & 0,06 & 16,56 & 3 \\
\hline 1991 & 169,70 & 2,32 & 0,91 & 0,09 & 10,66 & 4 \\
\hline 1984 & 162,60 & 2,06 & 0,88 & 0,12 & 8,35 & 5 \\
\hline 1993 & 159,00 & 1,93 & 0,86 & 0,14 & 7,39 & 6 \\
\hline 1998 & 157,00 & 1,86 & 0,86 & 0,14 & 6,91 & 7 \\
\hline 2007 & 151,40 & 1,65 & 0,83 & 0,17 & 5,73 & 8 \\
\hline 1980 & 148,30 & 1,54 & 0,81 & 0,19 & 5,17 & 9 \\
\hline 1990 & 147,50 & 1,51 & 0,80 & 0,20 & 5,04 & 10 \\
\hline 2009 & 146,40 & 1,47 & 0,79 & 0,21 & 4,86 & 11 \\
\hline 1987 & 144,20 & 1,39 & 0,78 & 0,22 & 4,53 & 12 \\
\hline 1985 & 142,50 & 1,33 & 0,77 & 0,23 & 4,29 & 13 \\
\hline 1988 & 139,50 & 1,22 & 0,74 & 0,26 & 3,90 & 14 \\
\hline 2002 & 139,00 & 1,20 & 0,74 & 0,26 & 3,84 & 15 \\
\hline 1961 & 137,70 & 1,15 & 0,73 & 0,27 & 3,69 & 16 \\
\hline 1964 & 135,20 & 1,06 & 0,71 & 0,29 & 3,42 & 17 \\
\hline 2001 & 131,00 & 0,91 & 0,67 & 0,33 & 3,01 & 18 \\
\hline 1977 & 130,70 & 0,90 & 0,67 & 0,33 & 2,99 & 19 \\
\hline 1975 & 130,00 & 0,87 & 0,66 & 0,34 & 2,93 & 20 \\
\hline 1982 & 126,90 & 0,76 & 0,63 & 0,37 & 2,68 & 21 \\
\hline 1983 & 124,80 & 0,68 & 0,60 & 0,40 & 2,52 & 22 \\
\hline 1974 & 124,40 & 0,67 & 0,60 & 0,40 & 2,49 & 23 \\
\hline 1969 & 120,60 & 0,53 & 0,56 & 0,44 & 2,25 & 24 \\
\hline 1978 & 120,30 & 0,52 & 0,55 & 0,45 & 2,23 & 25 \\
\hline 1972 & 118,70 & 0,46 & 0,53 & 0,47 & 2,14 & 26 \\
\hline 1996 & 116,20 & 0,37 & 0,50 & 0,50 & 2,00 & 27 \\
\hline 1994 & 115,00 & 0,33 & 0,49 & 0,51 & 1,94 & 28 \\
\hline 1973 & 113,20 & 0,26 & 0,46 & 0,54 & 1,86 & 29 \\
\hline 1976 & 111,80 & 0,21 & 0,44 & 0,56 & 1,80 & 30 \\
\hline
\end{tabular}




\begin{tabular}{|c|c|c|c|c|c|c|}
\hline 2010 & 108,40 & 0,09 & 0,40 & 0,60 & 1,66 & 31 \\
\hline 1962 & 108,20 & 0,08 & 0,40 & 0,60 & 1,66 & 32 \\
\hline 2003 & 105,30 & $-0,03$ & 0,36 & 0,64 & 1,56 & 33 \\
\hline 1971 & 103,20 & $-0,10$ & 0,33 & 0,67 & 1,49 & 34 \\
\hline 1981 & 102,30 & $-0,14$ & 0,32 & 0,68 & 1,47 & 35 \\
\hline 1965 & 102,00 & $-0,15$ & 0,31 & 0,69 & 1,46 & 36 \\
\hline 2005 & 100,40 & $-0,21$ & 0,29 & 0,71 & 1,41 & 37 \\
\hline 1963 & 94,80 & $-0,41$ & 0,22 & 0,78 & 1,28 & 38 \\
\hline 1995 & 94,80 & $-0,41$ & 0,22 & 0,78 & 1,28 & 39 \\
\hline 2000 & 94,20 & $-0,43$ & 0,21 & 0,79 & 1,27 & 40 \\
\hline 1986 & 91,80 & $-0,52$ & 0,19 & 0,81 & 1,23 & 41 \\
\hline 1970 & 90,60 & $-0,56$ & 0,17 & 0,83 & 1,21 & 42 \\
\hline 2004 & 88,60 & $-0,64$ & 0,15 & 0,85 & 1,18 & 43 \\
\hline 1968 & 87,90 & $-0,66$ & 0,14 & 0,86 & 1,17 & 44 \\
\hline 1999 & 85,30 & $-0,76$ & 0,12 & 0,88 & 1,14 & 45 \\
\hline 1966 & 81,70 & $-0,89$ & 0,09 & 0,91 & 1,10 & 46 \\
\hline 1979 & 79,40 & $-0,97$ & 0,07 & 0,93 & 1,08 & 47 \\
\hline 1967 & 69,80 & $-1,32$ & 0,02 & 0,98 & 1,02 & 48 \\
\hline 2006 & 63,00 & $-1,57$ & 0,01 & 0,99 & 1,01 & 49 \\
\hline 2008 & 62,80 & $-1,57$ & 0,01 & 0,99 & 1,01 & 50 \\
\hline MÉDIA (x) & 121,16 & & & & & \\
\hline DESVIO & & & & & & \\
\hline $\begin{array}{l}\text { PADRÃO } \\
(\text { (Sx) }\end{array}$ & 31,86 & & & & & \\
\hline Sn & 1,16 & & & & & \\
\hline Yn & 0,55 & & & & & \\
\hline$X f$ & 106,056 & & & & & \\
\hline
\end{tabular}

TABELA 4 - VALORES OBTIDOS PARA PRECIPITAÇÕES MÁXIMAS COM DURAÇÃO DE 3 DIAS

\begin{tabular}{ccccccc}
\hline ANO & $\begin{array}{c}\text { Precipitação } \\
\text { em 3 dias }\end{array}$ & $\mathbf{Y}$ & $\mathbf{P}$ & $\mathbf{( 1 - P )}$ & $\begin{array}{c}\text { Período de } \\
\text { Recorrência } \\
\text { em anos (T) }\end{array}$ & $\begin{array}{c}\text { Ordem: } \\
\text { 3 dias (r) }\end{array}$ \\
\hline $\mathbf{1 9 8 9}$ & 264,00 & 4,41 & 0,99 & 0,01 & 83,03 & 1 \\
$\mathbf{1 9 9 7}$ & 205,70 & 2,58 & 0,93 & 0,07 & 13,71 & 2 \\
$\mathbf{2 0 0 2}$ & 197,30 & 2,32 & 0,91 & 0,09 & 10,65 & 3 \\
$\mathbf{1 9 8 0}$ & 195,10 & 2,25 & 0,90 & 0,10 & 9,97 & 4 \\
$\mathbf{1 9 9 1}$ & 193,30 & 2,19 & 0,89 & 0,11 & 9,45 & 5 \\
$\mathbf{1 9 9 3}$ & 192,20 & 2,16 & 0,89 & 0,11 & 9,15 & 6 \\
$\mathbf{1 9 9 2}$ & 185,40 & 1,94 & 0,87 & 0,13 & 7,49 & 7 \\
$\mathbf{1 9 6 4}$ & 174,00 & 1,58 & 0,81 & 0,19 & 5,39 & 8 \\
$\mathbf{1 9 7 7}$ & 172,50 & 1,54 & 0,81 & 0,19 & 5,17 & 9 \\
$\mathbf{1 9 9 0}$ & 172,20 & 1,53 & 0,80 & 0,20 & 5,13 & 10 \\
$\mathbf{2 0 0 9}$ & 168,00 & 1,40 & 0,78 & 0,22 & 4,56 & 11 \\
$\mathbf{1 9 9 8}$ & 166,40 & 1,35 & 0,77 & 0,23 & 4,36 & 12 \\
$\mathbf{1 9 8 4}$ & 166,10 & 1,34 & 0,77 & 0,23 & 4,33 & 13 \\
$\mathbf{1 9 7 4}$ & 163,80 & 1,26 & 0,75 & 0,25 & 4,06 & 14 \\
$\mathbf{1 9 8 7}$ & 159,60 & 1,13 & 0,72 & 0,28 & 3,63 & 15 \\
\hline
\end{tabular}




\begin{tabular}{|c|c|c|c|c|c|c|}
\hline 2007 & 156,80 & 1,04 & 0,70 & 0,30 & 3,37 & 16 \\
\hline 1983 & 156,00 & 1,02 & 0,70 & 0,30 & 3,30 & 17 \\
\hline 1961 & 147,90 & 0,76 & 0,63 & 0,37 & 2,69 & 18 \\
\hline 2001 & 146,80 & 0,73 & 0,62 & 0,38 & 2,61 & 19 \\
\hline 1975 & 146,20 & 0,71 & 0,61 & 0,39 & 2,58 & 20 \\
\hline 1965 & 144,20 & 0,65 & 0,59 & 0,41 & 2,46 & 21 \\
\hline 1985 & 142,50 & 0,59 & 0,58 & 0,42 & 2,36 & 22 \\
\hline 1976 & 141,60 & 0,57 & 0,57 & 0,43 & 2,31 & 23 \\
\hline 1973 & 141,40 & 0,56 & 0,56 & 0,44 & 2,30 & 24 \\
\hline 1988 & 139,90 & 0,51 & 0,55 & 0,45 & 2,22 & 25 \\
\hline 2003 & 138,00 & 0,45 & 0,53 & 0,47 & 2,13 & 26 \\
\hline 1996 & 137,80 & 0,45 & 0,53 & 0,47 & 2,12 & 27 \\
\hline 1982 & 136,30 & 0,40 & 0,51 & 0,49 & 2,05 & 28 \\
\hline 2005 & 135,50 & 0,37 & 0,50 & 0,50 & 2,01 & 29 \\
\hline 1978 & 134,80 & 0,35 & 0,50 & 0,50 & 1,98 & 30 \\
\hline 1981 & 131,90 & 0,26 & 0,46 & 0,54 & 1,86 & 31 \\
\hline 1994 & 128,30 & 0,15 & 0,42 & 0,58 & 1,73 & 32 \\
\hline 1995 & 121,90 & $-0,05$ & 0,35 & 0,65 & 1,53 & 33 \\
\hline 1971 & 121,50 & $-0,07$ & 0,34 & 0,66 & 1,52 & 34 \\
\hline 1969 & 120,60 & $-0,09$ & 0,33 & 0,67 & 1,50 & 35 \\
\hline 1970 & 120,60 & $-0,09$ & 0,33 & 0,67 & 1,50 & 36 \\
\hline 1972 & 118,70 & $-0,15$ & 0,31 & 0,69 & 1,45 & 37 \\
\hline 2010 & 117,40 & $-0,19$ & 0,30 & 0,70 & 1,42 & 38 \\
\hline 1962 & 114,60 & $-0,28$ & 0,27 & 0,73 & 1,36 & 39 \\
\hline 2004 & 111,10 & $-0,39$ & 0,23 & 0,77 & 1,29 & 40 \\
\hline 2006 & 107,50 & $-0,51$ & 0,19 & 0,81 & 1,24 & 41 \\
\hline 2000 & 102,70 & $-0,66$ & 0,15 & 0,85 & 1,17 & 42 \\
\hline 1963 & 101,00 & $-0,71$ & 0,13 & 0,87 & 1,15 & 43 \\
\hline 1999 & 97,40 & $-0,82$ & 0,10 & 0,90 & 1,11 & 44 \\
\hline 2008 & 96,20 & $-0,86$ & 0,09 & 0,91 & 1,10 & 45 \\
\hline 1966 & 90,90 & $-1,03$ & 0,06 & 0,94 & 1,07 & 46 \\
\hline 1967 & 86,80 & $-1,16$ & 0,04 & 0,96 & 1,04 & 47 \\
\hline 1968 & 85,50 & $-1,20$ & 0,04 & 0,96 & 1,04 & 48 \\
\hline 1979 & 79,40 & $-1,39$ & 0,02 & 0,98 & 1,02 & 49 \\
\hline 1986 & 79,00 & $-1,40$ & 0,02 & 0,98 & 1,02 & 50 \\
\hline $\begin{array}{l}\text { MÉDIA } \\
(x)\end{array}$ & 141,09 & & & & & \\
\hline DESVIO & & & & & & \\
\hline $\begin{array}{l}\text { PADRÃO } \\
\text { (Sx) }\end{array}$ & 36,91 & & & & & \\
\hline Sn & 1,16 & & & & & \\
\hline Yn & 0,55 & & & & & \\
\hline$X f$ & 123,588 & & & & & \\
\hline
\end{tabular}


TABELA 5 - VALORES OBTIDOS PARA PRECIPITAÇÕES MÁXIMAS COM DURAÇÃO DE 4 DIAS

\begin{tabular}{|c|c|c|c|c|c|c|}
\hline ANO & $\begin{array}{l}\text { Precipitação } \\
\text { em } 4 \text { dias }\end{array}$ & $\mathbf{Y}$ & $\mathbf{P}$ & $(1-P)$ & $\begin{array}{l}\text { Período de } \\
\text { Recorrência } \\
\text { em anos }(T)\end{array}$ & $\begin{array}{l}\text { Ordem: } \\
4 \text { dias (r) }\end{array}$ \\
\hline 1989 & 291,50 & 4,41 & 0,99 & 0,01 & 82,47 & 1 \\
\hline 1997 & 226,50 & 2,55 & 0,92 & 0,08 & 13,31 & 2 \\
\hline 2002 & 217,80 & 2,30 & 0,90 & 0,10 & 10,49 & 3 \\
\hline 1980 & 218,50 & 2,32 & 0,91 & 0,09 & 10,70 & 4 \\
\hline 1991 & 193,30 & 1,60 & 0,82 & 0,18 & 5,48 & 5 \\
\hline 1993 & 219,20 & 2,34 & 0,91 & 0,09 & 10,90 & 6 \\
\hline 1992 & 190,40 & 1,52 & 0,80 & 0,20 & 5,08 & 7 \\
\hline 1964 & 191,00 & 1,54 & 0,81 & 0,19 & 5,16 & 8 \\
\hline 1977 & 174,90 & 1,08 & 0,71 & 0,29 & 3,46 & 9 \\
\hline 1990 & 267,50 & 3,72 & 0,98 & 0,02 & 41,80 & 10 \\
\hline 2009 & 182,60 & 1,30 & 0,76 & 0,24 & 4,18 & 11 \\
\hline 1998 & 175,50 & 1,09 & 0,72 & 0,28 & 3,51 & 12 \\
\hline 1984 & 166,10 & 0,82 & 0,64 & 0,36 & 2,82 & 13 \\
\hline 1974 & 168,10 & 0,88 & 0,66 & 0,34 & 2,95 & 14 \\
\hline 1987 & 160,40 & 0,66 & 0,60 & 0,40 & 2,48 & 15 \\
\hline 2007 & 156,80 & 0,56 & 0,56 & 0,44 & 2,30 & 16 \\
\hline 1983 & 193,80 & 1,62 & 0,82 & 0,18 & 5,55 & 17 \\
\hline 1961 & 149,80 & 0,36 & 0,50 & 0,50 & 1,99 & 18 \\
\hline 2001 & 146,80 & 0,27 & 0,47 & 0,53 & 1,88 & 19 \\
\hline 1975 & 156,80 & 0,56 & 0,56 & 0,44 & 2,30 & 20 \\
\hline 1965 & 145,40 & 0,23 & 0,45 & 0,55 & 1,83 & 21 \\
\hline 1985 & 142,50 & 0,15 & 0,42 & 0,58 & 1,73 & 22 \\
\hline 1976 & 147,20 & 0,28 & 0,47 & 0,53 & 1,89 & 23 \\
\hline 1973 & 156,20 & 0,54 & 0,56 & 0,44 & 2,27 & 24 \\
\hline 1988 & 140,60 & 0,10 & 0,40 & 0,60 & 1,68 & 25 \\
\hline 2003 & 166,70 & 0,84 & 0,65 & 0,35 & 2,86 & 26 \\
\hline 1996 & 157,90 & 0,59 & 0,57 & 0,43 & 2,35 & 27 \\
\hline 1982 & 143,10 & 0,17 & 0,43 & 0,57 & 1,75 & 28 \\
\hline 2005 & 155,50 & 0,52 & 0,55 & 0,45 & 2,23 & 29 \\
\hline 1978 & 138,20 & 0,03 & 0,38 & 0,62 & 1,61 & 30 \\
\hline 1981 & 139,90 & 0,08 & 0,40 & 0,60 & 1,66 & 31 \\
\hline 1994 & 128,40 & $-0,25$ & 0,28 & 0,72 & 1,38 & 32 \\
\hline 1995 & 148,10 & 0,31 & 0,48 & 0,52 & 1,92 & 33 \\
\hline 1971 & 128,10 & $-0,26$ & 0,27 & 0,73 & 1,38 & 34 \\
\hline 1969 & 120,60 & $-0,48$ & 0,20 & 0,80 & 1,25 & 35 \\
\hline 1970 & 128,50 & $-0,25$ & 0,28 & 0,72 & 1,38 & 36 \\
\hline 1972 & 166,00 & 0,82 & 0,64 & 0,36 & 2,81 & 37 \\
\hline 2010 & 130,00 & $-0,21$ & 0,29 & 0,71 & 1,41 & 38 \\
\hline 1962 & 122,00 & $-0,44$ & 0,21 & 0,79 & 1,27 & 39 \\
\hline 2004 & 122,80 & $-0,41$ & 0,22 & 0,78 & 1,28 & 40 \\
\hline 2006 & 139,80 & 0,07 & 0,39 & 0,61 & 1,65 & 41 \\
\hline 2000 & 121,80 & $-0,44$ & 0,21 & 0,79 & 1,27 & 42 \\
\hline 1963 & 118,70 & $-0,53$ & 0,18 & 0,82 & 1,22 & 43 \\
\hline 1999 & 127,20 & $-0,29$ & 0,26 & 0,74 & 1,36 & 44 \\
\hline 2008 & 96,20 & $-1,17$ & 0,04 & 0,96 & 1,04 & 45 \\
\hline
\end{tabular}




\begin{tabular}{ccccccc}
\hline $\mathbf{1 9 6 6}$ & 91,90 & $-1,30$ & 0,03 & 0,97 & 1,03 & 46 \\
$\mathbf{1 9 6 7}$ & 87,30 & $-1,43$ & 0,02 & 0,98 & 1,02 & 47 \\
$\mathbf{1 9 6 8}$ & 135,50 & $-0,05$ & 0,35 & 0,65 & 1,54 & 48 \\
$\mathbf{1 9 7 9}$ & 123,30 & $-0,40$ & 0,23 & 0,77 & 1,29 & 49 \\
$\mathbf{1 9 8 6}$ & 108,30 & $-0,83$ & 0,10 & 0,90 & 1,11 & 50 \\
\hline MÉDIA & 156,50 & & & & & \\
$\mathbf{( x )}$ & & & & & & \\
DESVIO & & & & & & \\
PADRÃO & 40,61 & & & & & \\
$\mathbf{( S \mathbf { X } )}$ & & & & & \\
$\mathbf{S n}$ & 1,16 & & & & & \\
Yn & 0,55 & & & & & \\
$\mathbf{X f}$ & 137,243 & & & & & \\
\hline
\end{tabular}

TABELA 6 - VALORES OBTIDOS PARA PRECIPITAÇÕES MÁXIMAS COM DURAÇÃO DE 5 DIAS

\begin{tabular}{|c|c|c|c|c|c|c|}
\hline ANO & $\begin{array}{c}\text { Precipitação } \\
\text { em } 5 \text { dias }\end{array}$ & $\mathbf{Y}$ & $\mathbf{P}$ & $(1-P)$ & $\begin{array}{l}\text { Período de } \\
\text { Recorrência } \\
\text { em anos }(T)\end{array}$ & $\begin{array}{l}\text { Ordem: } \\
5 \text { dias(r) }\end{array}$ \\
\hline 1989 & 308,30 & 4,12 & 0,98 & 0,02 & 62,22 & 1 \\
\hline 1990 & 295,00 & 3,78 & 0,98 & 0,02 & 44,43 & 2 \\
\hline 1980 & 249,50 & 2,62 & 0,93 & 0,07 & 14,24 & 3 \\
\hline 1997 & 243,50 & 2,47 & 0,92 & 0,08 & 12,28 & 4 \\
\hline 2002 & 233,60 & 2,21 & 0,90 & 0,10 & 9,65 & 5 \\
\hline 1993 & 225,80 & 2,01 & 0,88 & 0,12 & 8,00 & 6 \\
\hline 1964 & 218,70 & 1,83 & 0,85 & 0,15 & 6,76 & 7 \\
\hline 2009 & 216,40 & 1,77 & 0,84 & 0,16 & 6,41 & 8 \\
\hline 1992 & 210,70 & 1,63 & 0,82 & 0,18 & 5,61 & 9 \\
\hline 2003 & 204,00 & 1,46 & 0,79 & 0,21 & 4,81 & 10 \\
\hline 1974 & 199,90 & 1,35 & 0,77 & 0,23 & 4,39 & 11 \\
\hline 1972 & 199,50 & 1,34 & 0,77 & 0,23 & 4,35 & 12 \\
\hline 1983 & 194,10 & 1,20 & 0,74 & 0,26 & 3,86 & 13 \\
\hline 1991 & 193,30 & 1,18 & 0,74 & 0,26 & 3,79 & 14 \\
\hline 2005 & 185,80 & 0,99 & 0,69 & 0,31 & 3,23 & 15 \\
\hline 1998 & 184,90 & 0,97 & 0,68 & 0,32 & 3,16 & 16 \\
\hline 1968 & 182,30 & 0,90 & 0,67 & 0,33 & 3,00 & 17 \\
\hline 1977 & 175,30 & 0,72 & 0,62 & 0,38 & 2,60 & 18 \\
\hline 1973 & 170,40 & 0,60 & 0,58 & 0,42 & 2,36 & 19 \\
\hline 1996 & 166,90 & 0,51 & 0,55 & 0,45 & 2,21 & 20 \\
\hline 1984 & 166,10 & 0,49 & 0,54 & 0,46 & 2,18 & 21 \\
\hline 2007 & 162,90 & 0,41 & 0,51 & 0,49 & 2,06 & 22 \\
\hline 1969 & 161,00 & 0,36 & 0,50 & 0,50 & 1,99 & 23 \\
\hline 1987 & 160,40 & 0,34 & 0,49 & 0,51 & 1,97 & 24 \\
\hline 1995 & 159,50 & 0,32 & 0,48 & 0,52 & 1,94 & 25 \\
\hline 1975 & 156,80 & 0,25 & 0,46 & 0,54 & 1,85 & 26 \\
\hline 1965 & 150,30 & 0,08 & 0,40 & 0,60 & 1,66 & 27 \\
\hline 1961 & 149,80 & 0,07 & 0,39 & 0,61 & 1,65 & 28 \\
\hline 2006 & 147,80 & 0,02 & 0,38 & 0,62 & 1,60 & 29 \\
\hline
\end{tabular}




\begin{tabular}{|c|c|c|c|c|c|c|}
\hline 1976 & 147,20 & 0,00 & 0,37 & 0,63 & 1,59 & 30 \\
\hline 2001 & 146,80 & $-0,01$ & 0,37 & 0,63 & 1,58 & 31 \\
\hline 1982 & 144,40 & $-0,07$ & 0,34 & 0,66 & 1,52 & 32 \\
\hline 1994 & 143,10 & $-0,10$ & 0,33 & 0,67 & 1,49 & 33 \\
\hline 1985 & 142,50 & $-0,12$ & 0,33 & 0,67 & 1,48 & 34 \\
\hline 1970 & 142,00 & $-0,13$ & 0,32 & 0,68 & 1,47 & 35 \\
\hline 1971 & 141,10 & $-0,15$ & 0,31 & 0,69 & 1,45 & 36 \\
\hline 1988 & 140,60 & $-0,16$ & 0,31 & 0,69 & 1,44 & 37 \\
\hline 1981 & 140,50 & $-0,17$ & 0,31 & 0,69 & 1,44 & 38 \\
\hline 1978 & 138,50 & $-0,22$ & 0,29 & 0,71 & 1,41 & 39 \\
\hline 2010 & 137,60 & $-0,24$ & 0,28 & 0,72 & 1,39 & 40 \\
\hline 1963 & 136,40 & $-0,27$ & 0,27 & 0,73 & 1,37 & 41 \\
\hline 2004 & 135,60 & $-0,29$ & 0,26 & 0,74 & 1,36 & 42 \\
\hline 1999 & 129,90 & $-0,44$ & 0,21 & 0,79 & 1,27 & 43 \\
\hline 1979 & 128,30 & $-0,48$ & 0,20 & 0,80 & 1,25 & 44 \\
\hline 1962 & 126,40 & $-0,53$ & 0,18 & 0,82 & 1,23 & 45 \\
\hline 2000 & 121,80 & $-0,64$ & 0,15 & 0,85 & 1,17 & 46 \\
\hline 1986 & 113,30 & $-0,86$ & 0,09 & 0,91 & 1,10 & 47 \\
\hline 2008 & 107,90 & $-1,00$ & 0,07 & 0,93 & 1,07 & 48 \\
\hline 1967 & 97,50 & $-1,27$ & 0,03 & 0,97 & 1,03 & 49 \\
\hline 1966 & 93,00 & $-1,38$ & 0,02 & 0,98 & 1,02 & 50 \\
\hline MÉDIA (x) & 168,54 & & & & & \\
\hline DESVIO & & & & & & \\
\hline $\begin{array}{l}\text { PADRÃO } \\
\text { (Sx) }\end{array}$ & 45,38 & & & & & \\
\hline Sn & 1,16 & & & & & \\
\hline Yn & 0,55 & & & & & \\
\hline$X f$ & 147,023 & & & & & \\
\hline
\end{tabular}

TABELA 7 - VALORES OBTIDOS PARA PRECIPITAÇÕES MÁXIMAS COM DURAÇÃO DE 6 DIAS

\begin{tabular}{ccccccc}
\hline ANO & $\begin{array}{c}\text { Precipitação } \\
\text { em6 dias }\end{array}$ & $\mathbf{Y}$ & $\mathbf{P}$ & $\mathbf{( 1 - P )}$ & $\begin{array}{c}\text { Período de } \\
\text { Recorrência } \\
\mathbf{( T )}\end{array}$ & $\begin{array}{c}\text { Ordem: } \\
\mathbf{6} \text { dias (r) }\end{array}$ \\
\hline $\mathbf{1 9 9 0}$ & 311,80 & 3,76 & 0,98 & 0,02 & 43,25 & 1 \\
$\mathbf{1 9 8 9}$ & 308,30 & 3,67 & 0,97 & 0,03 & 39,82 & 2 \\
$\mathbf{1 9 8 0}$ & 266,90 & 2,68 & 0,93 & 0,07 & 15,11 & 3 \\
$\mathbf{2 0 0 9}$ & 266,80 & 2,68 & 0,93 & 0,07 & 15,07 & 4 \\
$\mathbf{1 9 9 7}$ & 246,50 & 2,19 & 0,89 & 0,11 & 9,47 & 5 \\
$\mathbf{2 0 0 2}$ & 238,60 & 2,00 & 0,87 & 0,13 & 7,93 & 6 \\
$\mathbf{1 9 6 4}$ & 236,20 & 1,95 & 0,87 & 0,13 & 7,52 & 7 \\
$\mathbf{1 9 9 3}$ & 227,10 & 1,73 & 0,84 & 0,16 & 6,15 & 8 \\
$\mathbf{2 0 0 3}$ & 225,30 & 1,69 & 0,83 & 0,17 & 5,91 & 9 \\
$\mathbf{2 0 0 5}$ & 220,90 & 1,58 & 0,81 & 0,19 & 5,38 & 10 \\
$\mathbf{1 9 9 2}$ & 210,70 & 1,34 & 0,77 & 0,23 & 4,33 & 11 \\
$\mathbf{1 9 7 4}$ & 204,70 & 1,19 & 0,74 & 0,26 & 3,82 & 12 \\
$\mathbf{2 0 0 7}$ & 203,40 & 1,16 & 0,73 & 0,27 & 3,72 & 13 \\
$\mathbf{1 9 8 3}$ & 201,80 & 1,12 & 0,72 & 0,28 & 3,60 & 14 \\
\hline
\end{tabular}




\begin{tabular}{|c|c|c|c|c|c|c|}
\hline 1972 & 200,90 & 1,10 & 0,72 & 0,28 & 3,54 & 15 \\
\hline 1968 & 200,60 & 1,10 & 0,72 & 0,28 & 3,52 & 16 \\
\hline 1991 & 193,30 & 0,92 & 0,67 & 0,33 & 3,04 & 17 \\
\hline 1984 & 186,90 & 0,77 & 0,63 & 0,37 & 2,69 & 18 \\
\hline 1998 & 185,10 & 0,72 & 0,62 & 0,38 & 2,60 & 19 \\
\hline 1973 & 181,80 & 0,65 & 0,59 & 0,41 & 2,45 & 20 \\
\hline 1975 & 181,40 & 0,64 & 0,59 & 0,41 & 2,43 & 21 \\
\hline 1969 & 175,70 & 0,50 & 0,55 & 0,45 & 2,20 & 22 \\
\hline 1977 & 175,30 & 0,49 & 0,54 & 0,46 & 2,18 & 23 \\
\hline 1996 & 170,50 & 0,38 & 0,50 & 0,50 & 2,01 & 24 \\
\hline 1995 & 169,00 & 0,34 & 0,49 & 0,51 & 1,96 & 25 \\
\hline 1970 & 162,90 & 0,19 & 0,44 & 0,56 & 1,78 & 26 \\
\hline 1978 & 160,60 & 0,14 & 0,42 & 0,58 & 1,72 & 27 \\
\hline 1987 & 160,40 & 0,13 & 0,42 & 0,58 & 1,71 & 28 \\
\hline 1965 & 159,50 & 0,11 & 0,41 & 0,59 & 1,69 & 29 \\
\hline 1971 & 157,00 & 0,05 & 0,39 & 0,61 & 1,63 & 30 \\
\hline 2001 & 153,70 & $-0,03$ & 0,36 & 0,64 & 1,56 & 31 \\
\hline 1961 & 153,20 & $-0,04$ & 0,35 & 0,65 & 1,55 & 32 \\
\hline 1994 & 150,10 & $-0,11$ & 0,33 & 0,67 & 1,48 & 33 \\
\hline 1982 & 148,40 & $-0,15$ & 0,31 & 0,69 & 1,45 & 34 \\
\hline 2006 & 147,80 & $-0,17$ & 0,31 & 0,69 & 1,44 & 35 \\
\hline 1976 & 147,20 & $-0,18$ & 0,30 & 0,70 & 1,43 & 36 \\
\hline 1985 & 145,10 & $-0,23$ & 0,28 & 0,72 & 1,39 & 37 \\
\hline 2010 & 144,00 & $-0,26$ & 0,27 & 0,73 & 1,38 & 38 \\
\hline 1963 & 143,50 & $-0,27$ & 0,27 & 0,73 & 1,37 & 39 \\
\hline 1988 & 143,40 & $-0,27$ & 0,27 & 0,73 & 1,37 & 40 \\
\hline 1981 & 140,50 & $-0,34$ & 0,24 & 0,76 & 1,32 & 41 \\
\hline 2004 & 136,20 & $-0,45$ & 0,21 & 0,79 & 1,27 & 42 \\
\hline 2000 & 136,00 & $-0,45$ & 0,21 & 0,79 & 1,26 & 43 \\
\hline 1999 & 134,10 & $-0,50$ & 0,19 & 0,81 & 1,24 & 44 \\
\hline 1979 & 129,10 & $-0,62$ & 0,16 & 0,84 & 1,19 & 45 \\
\hline 1962 & 126,90 & $-0,67$ & 0,14 & 0,86 & 1,17 & 46 \\
\hline 1986 & 114,00 & $-0,98$ & 0,07 & 0,93 & 1,08 & 47 \\
\hline 2008 & 109,20 & $-1,09$ & 0,05 & 0,95 & 1,05 & 48 \\
\hline 1967 & 101,20 & $-1,28$ & 0,03 & 0,97 & 1,03 & 49 \\
\hline 1966 & 97,20 & $-1,38$ & 0,02 & 0,98 & 1,02 & 50 \\
\hline MÉDIA (x) & 177,81 & & & & & \\
\hline DESVIO & & & & & & \\
\hline $\begin{array}{l}\text { PADRÃO } \\
(\mathrm{Sx})\end{array}$ & 48,49 & & & & & \\
\hline Sn & 1,16 & & & & & \\
\hline Yn & 0,55 & & & & & \\
\hline$X f$ & 154,824 & & & & & \\
\hline
\end{tabular}

A partir dos dados obtidos de precipitação, período de recorrência e do tempo de duração do evento pluvial, utilizou-se o software SigmaPlot para obtenção dos valores das variáveis para o ajuste à equação não linear de Holtz (1966), resultando na seguinte equação geral para Londrina: 
$H=85,4728 . T^{0,2345} \cdot(t-0,5521)^{0,2909}$

Assim, foi possível estimar as precipitações máximas esperadas para tempos de retorno $\mathbf{T}=2,5,10,20$ e 30 anos, com os devidos tempos de duração $\mathbf{t}(1$ a 6 dias) (Tabela 8). Os resultados podem ser visualizados na Figura 1.

TABELA 8 - PRECIPITAÇÕES MÁXIMAS ESTIMADAS PELA EQUAÇÃO GERAL DE HOLTZ PARA OS DIFERENTES PERÍODOS DE RECORRÊNCIA (T) COM DURAÇÕES DE 1 A 6 DIAS.

\begin{tabular}{rrrrrrr}
\hline $\begin{array}{l}\text { Anos de } \\
\text { Recorrência (T) }\end{array}$ & 1 dia & 2 dias & 3 dias & 4 dias & 5 dias & $\mathbf{6 ~ d i a s}$ \\
\hline $\mathbf{2}$ anos & 79,61 & 111,99 & 130,47 & 144,14 & 155,23 & 164,66 \\
\hline $\mathbf{5}$ anos & 98,69 & 138,83 & 161,75 & 178,69 & 192,44 & 204,13 \\
\hline $\mathbf{1 0}$ anos & 116,11 & 163,34 & 190,30 & 210,23 & 226,40 & 240,16 \\
\hline $\mathbf{2 0}$ anos & 136,60 & 192,17 & 223,88 & 247,34 & 266,36 & 282,55 \\
\hline 30 anos & 150,22 & 211,34 & 246,21 & 272,01 & 292,93 & 310,73
\end{tabular}

FIGURA 2 - CURVAS DE DURAÇÃO DE PRECIPITAÇÕES MÁXIMAS ESTIMADAS ATRAVÉS DA EQUAÇÃO GERAL DE HOLTZ PARA OS DIFERENTES PERÍODOS DE RECORRÊNCIA EM LONDRINA-PR

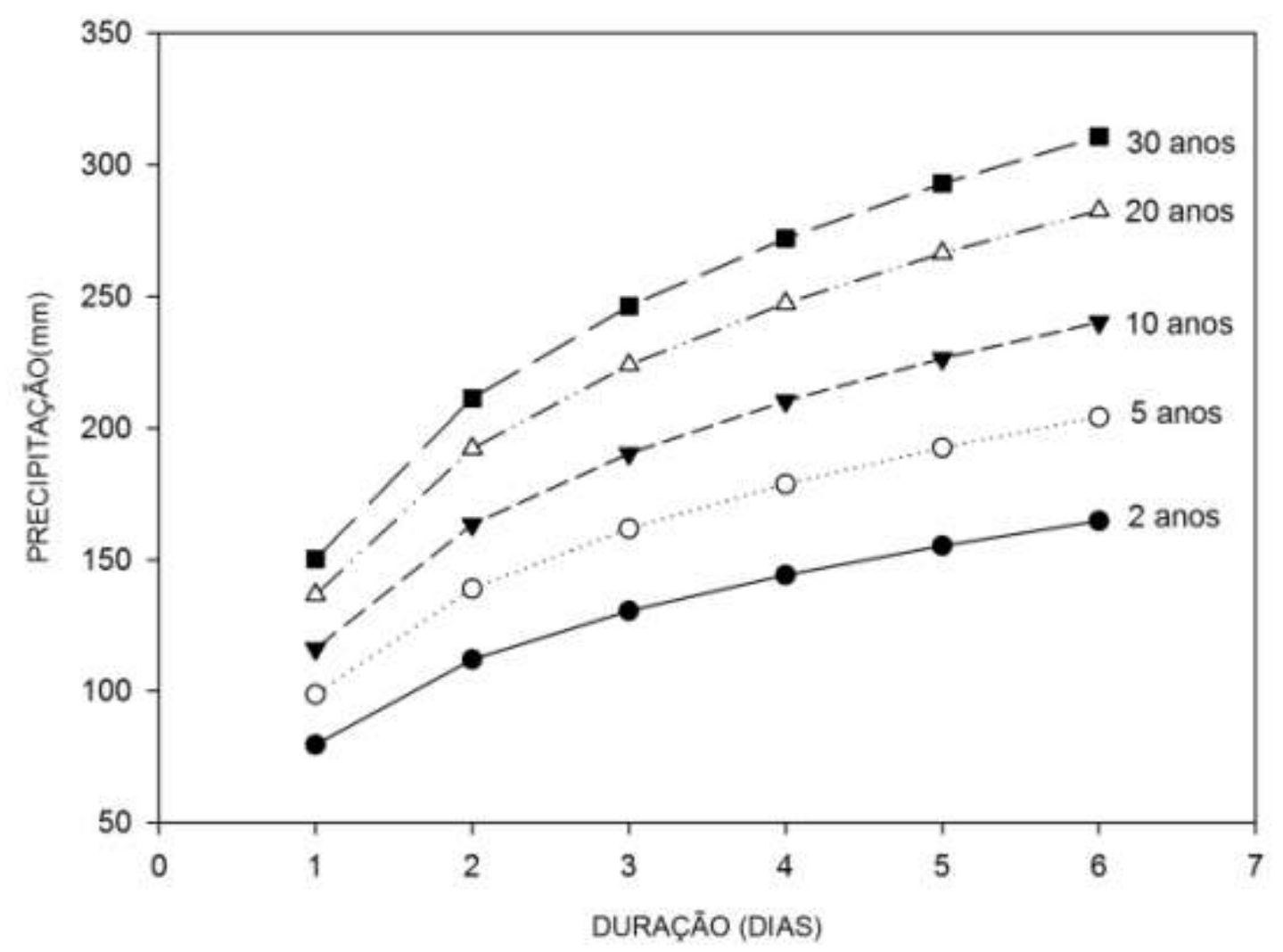


A figura 2 e a tabela 8 demonstram que conforme aumenta o tempo de vida da obra, maior será a probabilidade da mesma enfrentar maiores chuvas, sendo necessário considerar maior margem de segurança para que não haja comprometimentos por eventos pluviais maiores que sua capacidade de suporte.

A maior precipitação calculada foi de $310,73 \mathrm{~mm}$ acumulado em 6 dias para um período de 30 anos, mas como se trata de chuvas extremas, verifica-se que existe a probabilidade de ocorrer precipitações de 79,61 mm em apenas um dia para o período de retorno de 2 anos, um período de tempo curto com precipitações que podem gerar grandes danos tanto no meio urbano quanto no meio rural dependendo de sua intensidade.

É importante ressaltar que essas grandes quantidades pluviométricas registradas e calculadas podem estar ligadas a fenômenos climáticos que não foram totalmente desvendados pela ciência. Dentre os principais fenômenos de larga escala que afetam a precipitação, a ocorrência do El Niño - Oscilação Sul (ENOS) influencia em vários aspectos o clima local, regional e global (CPTEC, 2011).

Verificou-se que das 60 maiores precipitações (as 10 maiores em cada período de duração considerado, conforme tabelas 11 a 13), somente 10 (23,3\%), não foram registrados em anos de El Niño (EN) ou La Niña (LN) considerados fortes ou moderados, enquanto 55\% ocorreram em anos de EN e $21,5 \%$ em LN (CPTEC, 2011).

Considerando somente os anos presentes na relação, 33,3\% ocorreram em anos sem EN ou LN, 44,4\% em anos de EN e 22,2\% em anos de LN. Existe o predomínio das maiores pluviosidades em anos de El Niño, entretanto, anos sem a ocorrência do mesmo também apresentam fortes chuvas (Tabelas 9, 10 e 11).

O El Niño consiste no aumento anormal da temperatura do oceano Pacífico, enquanto a La Niña é o inverso. Pela alteração da temperatura do mar, altera-se e a dinâmica atmosférica e de correntes de ar, interferindo principalmente na distribuição de chuvas em parte do planeta. Já a Oscilação Sul diz respeito à diferença de pressão entre dois pontos, um localizado próximo à Indonésia e Norte da Austrália e outro próximo à costa da América do Sul, na ilha de Tahiti, gerando uma corrente de ar diferenciada.

Em estudos de Kousky e Cavalcanti (1984 apud NERY e FERREIRA, 1999), destacou-se que durante o fenômeno El Niño uma corrente de jato subtropical bem 
marcada sobre a América do Sul e a Oeste do Pacífico Sul juntamente com diversos bloqueios físicos explicariam as grandes pluviosidades na região sul, pois abriram espaço para a entrada de grandes frentes. Entretanto parte das maiores precipitações acumuladas não ocorreram em ano de El Niño. Isso pode ser justificado pelo fato de Londrina estar situada em zona de transição, diferenciando seu clima da maior parte da região Sul do Brasil. Além disso, alguns anos de La Niña,(que caracterizariam a região sul como período mais seco), também constam dentre os mais chuvosos, demonstrando que a dinâmica geral de distribuição de chuvas na região sul não se aplica inteiramente ao município de Londrina.

Ademais, segundo Nery e Ferreira (1999), nos mais fortes ENOS dos últimos anos que ocorreram em 1990 e 1997, foram registradas precipitações dentre as maiores da série histórica de Londrina. A análise rítmica dos anos desses episódios pode trazer maiores explicações quanto à influência desse fenômeno climático no município.

Em classificação mais detalhada da área, Silva et al.(2006) propõe um zoneamento climático de fronteira entre Mato Grosso do Sul, São Paulo e Paraná, abarcando Londrina. Nessa classificação, que leva em consideração diversas medições de condições climáticas, considera a zona de Londrina como "área controlada pela alternância de sistemas tropicais e polares e sob forte influência de sistemas individualizados, área de clima úmido e com as maiores precipitações pluviométricas de abril a novembro" (SILVA, 2006, p. 67), demonstrando a alternância de frentes e condições climáticas locais que influenciam a dinâmica no município e toda a zona de transição.

TABELA 9 - FENÔMENO EL NIÑO E LA NIÑA CLASSIFICADOS ACIMA DE MODERADO PREDOMINANTES NOS ANOS DE OCORRÊNCIA DOS EVENTOS

\begin{tabular}{lll|lll}
\hline \multicolumn{2}{l|}{ Precipitação em 1 dia } & & \multicolumn{3}{l}{ Acumulado de precipitação 2 dias } \\
\hline ANO & PRECIPITAÇÃO $(\mathrm{mm})$ & Fenômeno no ano & ANO & PRECIPITAÇÃO $(\mathrm{mm})$ & Fenômeno no ano \\
$\mathbf{1 9 9 7}$ & 161,0 & El Niño & $\mathbf{1 9 8 9}$ & 212,4 & La Niña \\
$\mathbf{1 9 8 4}$ & 151,2 & - & $\mathbf{1 9 9 2}$ & 185,2 & El Niño \\
$\mathbf{1 9 9 2}$ & 142,7 & El Niño & $\mathbf{1 9 9 7}$ & 182,3 & El Niño \\
$\mathbf{1 9 9 1}$ & 119,1 & El Niño & $\mathbf{1 9 9 1}$ & 169,7 & El Niño \\
$\mathbf{1 9 8 9}$ & 117,1 & La Niña & $\mathbf{1 9 8 4}$ & 162,6 & - \\
$\mathbf{1 9 7 7}$ & 116,3 & - & $\mathbf{1 9 9 3}$ & 159 & El Niño \\
$\mathbf{1 9 9 3}$ & 113,5 & El Niño & $\mathbf{1 9 9 8}$ & 157 & La Niña \\
\hline
\end{tabular}




\begin{tabular}{lll|lll}
\hline $\mathbf{2 0 1 0}$ & 108,2 & - & $\mathbf{2 0 0 7}$ & 151,4 & La Niña \\
$\mathbf{1 9 9 4}$ & 106,4 & El Niño & $\mathbf{1 9 8 0}$ & 148,3 & - \\
$\mathbf{2 0 0 7}$ & 104,8 & La Niña & $\mathbf{1 9 9 0}$ & 147,5 & El Niño \\
\hline
\end{tabular}

TABELA 10 - FENÔMENO EL NIÑO E LA NIÑA CLASSIFICADOS ACIMA DE MODERADO PREDOMINANTES NOS ANOS DE OCORRÊNCIA DOS EVENTOS.

\begin{tabular}{lll|lll}
\hline \multicolumn{3}{l}{ Acumulado de precipitação 3 dias } & \multicolumn{3}{l}{ Acumulado de precipitação 4 dias } \\
\hline ANO & PRECIPITAÇÃO $(\mathrm{mm})$ & Fenômeno no ano & ANO & PRECIPITAÇÃO $(\mathrm{mm})$ & Fenômeno no ano \\
$\mathbf{1 9 8 9}$ & 264 & La Niña & $\mathbf{1 9 8 9}$ & 291,5 & La Niña \\
$\mathbf{1 9 9 7}$ & 205,7 & El Niño & $\mathbf{1 9 9 7}$ & 226,5 & El Niño \\
$\mathbf{2 0 0 2}$ & 197,3 & El Niño & $\mathbf{2 0 0 2}$ & 217,8 & El Niño \\
$\mathbf{1 9 8 0}$ & 195,1 & - & $\mathbf{1 9 8 0}$ & 218,5 & - \\
$\mathbf{1 9 9 1}$ & 193,3 & El Niño & $\mathbf{1 9 9 1}$ & 193,3 & El Niño \\
$\mathbf{1 9 9 3}$ & 192,2 & El Niño & $\mathbf{1 9 9 3}$ & 219,2 & El Niño \\
$\mathbf{1 9 9 2}$ & 185,4 & El Niño & $\mathbf{1 9 9 2}$ & 190,4 & El Niño \\
$\mathbf{1 9 6 4}$ & 174 & La Niña & $\mathbf{1 9 6 4}$ & 191 & La Niña \\
$\mathbf{1 9 7 7}$ & 172,5 & - & $\mathbf{1 9 7 7}$ & 174,9 & - \\
$\mathbf{1 9 9 0}$ & 172,2 & El Niño & $\mathbf{1 9 9 0}$ & 267,5 & El Niño \\
\hline
\end{tabular}

TABELA 11 - FENÔMENO EL NIÑO E LA NIÑA CLASSIFICADOS ACIMA DE MODERADO PREDOMINANTES NOS ANOS DE OCORRÊNCIA DOS EVENTOS.

\begin{tabular}{lll|lll}
\hline \multicolumn{2}{l|}{ Acumulado de precipitação 5 dias } & \multicolumn{3}{l}{ Acumulado de precipitação 6 dias } \\
\hline ANO & PRECIPITAÇÃO (mm) & Fenômeno no ano & ANO & PRECIPITAÇÃO $(\mathrm{mm})$ & Fenômeno ano \\
$\mathbf{1 9 8 9}$ & 308,3 & La Niña & $\mathbf{1 9 9 0}$ & 311,8 & El Niño \\
1990 & 295 & El Niño & $\mathbf{1 9 8 9}$ & 308,3 & La Niña \\
1980 & 249,5 & - & $\mathbf{1 9 8 0}$ & 266,9 & - \\
1997 & 243,5 & El Niño & $\mathbf{2 0 0 9}$ & 266,8 & - \\
$\mathbf{2 0 0 2}$ & 233,6 & El Niño & $\mathbf{1 9 9 7}$ & 246,5 & El Niño \\
$\mathbf{1 9 9 3}$ & 225,8 & El Niño & $\mathbf{2 0 0 2}$ & 238,6 & El Niño \\
$\mathbf{1 9 6 4}$ & 218,7 & La Niña & $\mathbf{1 9 6 4}$ & 236,2 & La Niña \\
$\mathbf{2 0 0 9}$ & 216,4 & - & $\mathbf{1 9 9 3}$ & 227,1 & El Niño \\
$\mathbf{1 9 9 2}$ & 210,7 & El Niño & $\mathbf{2 0 0 3}$ & 225,3 & El Niño \\
$\mathbf{2 0 0 3}$ & 204 & El Niño & $\mathbf{2 0 0 5}$ & 220,9 & - \\
\hline
\end{tabular}




\section{CONCLUSÃO}

A utilização de técnicas estatísticas mostrou-se adequada para analisar a ocorrência de chuvas extremas no município de Londrina, fornecendo parâmetros adequados para dimensionar estruturas e contornar a ocorrência de desastres naturais. Com o ajuste de equações de período de retorno das chuvas extremas e de sua estimativa para diferentes períodos de duração existem elementos suficientes para a base do planejamento de edificações e estruturas nos meios rural e urbano.

Percebe-se que o ENOS exerce influência sobre o clima de Londrina, entretanto não se encontra um padrão para as chuvas, já que tanto em anos de La Niña quanto anos de El Niño existem registros de grandes precipitações. Por se situar um uma zona de transição climática, isso pode explicar os resultados constatados, demonstrando a grande diferença climática existente entre a Região Sul e o Norte Paranaense.

\section{REFERÊNCIAS}

CPTEC - Centro de Previsão de Tempo e Estudos Climáticos. Disponível em: http://enos.cptec.inpe.br/. Acesso em: 13 jan. 2012.

FARIA, R. T. de; WAGNER, C. S.Precipitações pluviais extremas no Paraná. Londrina: IAPAR, 1990.

MELLO, C.R. de; SILVA, A. M. da. Métodos estimadores dos parâmetros da distribuição de gumbel sua influência em estudos hidrológicos de projeto. Revista Irriga, Botucatu, v. 10, n. 4, p. 318-334, novembro-dezembro, 2005.

NAGHETTINI, M.; PINTO, E. J. A. Hidrologia Estatística. Belo Horizonte: CPRM, 2007.

NERY, J. T.; FERREIRA, J. H. D. Relação dos parâmetros meteorológicos associados com índice da Oscilação Sul. Boletim de Geografia, n. 17, p. 103-114, 1999.

SANT'ANNA NETO, J. L. Desmistificando o aquecimento global. 2007. Disponível em: http://www.awmueller.com/deposito/molion desmist.pdf. Acesso em: 16 jan. 2012.

SILVA, C. B. da; SANT'ANNA NETO, J. L; TOMMASELLI, J. T. G; PASSOS, M. M. dos. Dinâmica Atmosférica e Análise Geoestatística do Clima da Área de Integração 
Paisagística "Raia Divisória" Sp/Pr/Ms: Uma Proposta de Tipologia Climática. Revista Brasileira de Climatologia, v. 2, p. 53-70.

TUCCI, C. E. M. (org.) Hidrologia: ciência e aplicação. ABRH, Porto Alegre: Editora da UFRGS, 2000.

WMO - World Meteorological Organization. Guide to climatological practices. Genebra: WMO, 1983. 198p.

(Recebido em: 15/02/2012. Aceito em 06/12/2012). 